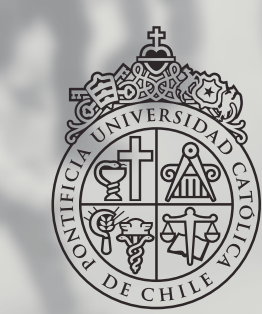

PONTIFICIA

UNIVERSIDAD

CATÓLICA

DE CHILE
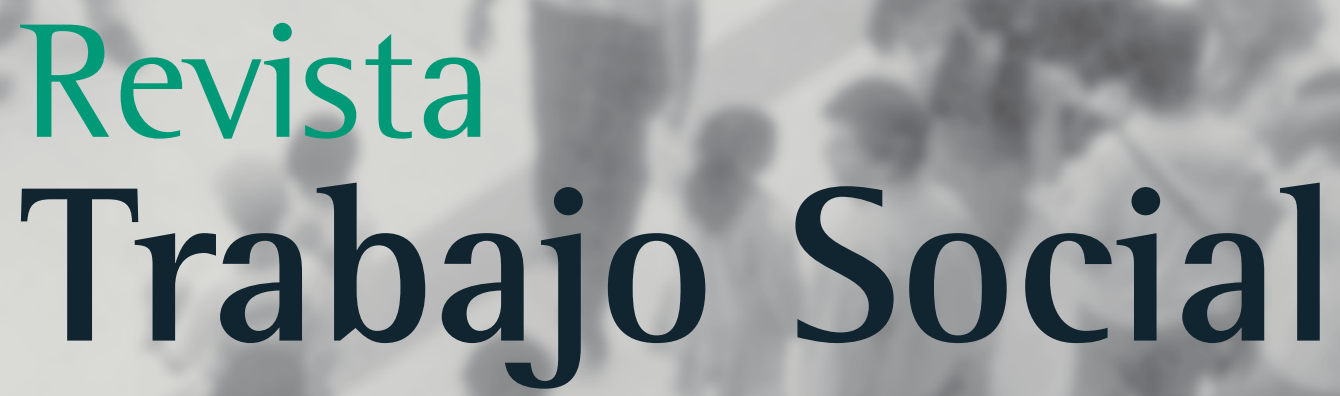

Intervención

socioambiental:

intersecciones del

Trabajo Social y la

institucionalidad

ambiental

Dr. Nelson Arellano-Escudero.

\section{Tensiones en la} Intervención Social:

(des)encuentros en la relación

Estado-ONG. Estudio de caso

sobre ONG que opera la política

social de infancia

Mauricio Sánchez Aliaga,

Rosa Villarroel ValdÉs.
Calidad de la participación infantil en la Política Local de Infancia Chilena

Daniela Díaz Bórquez,

Natalia Bozo Carrillo, Nicolás Contreras SHATS.

\section{Identidades Subyugadas}

entre Actores del Proceso

Penitenciario de Valparaíso

Paula Mercado Amin,

Fernanda Zavala Ossandón.

\section{Involucramiento}

laboral

y disposición al cambio

organizacional en el sector

de salud primaria

Carla Flores Figueroa,

LORENA MUÑoz MADRID,

Julia Jaramillo de Palacio. 



\title{
Intervención socioambiental: intersecciones del Trabajo Social y la institucionalidad ambiental
}

\section{Socio-environmental intervention: Intersections between Social Work and Environmental institutionality.}

\author{
Dr. Nelson Arellano-Escudero \\ Investigador responsable proyecto postdoctoral Fondecyt 3160197, Universidad de Tarapacá. Mail: narellano.5@gmail.com
}

\begin{abstract}
Resumen
La institucionalidad ambiental tiene carácter multidisciplinario y así concibe su misión de proteger el medio ambiente de manera de no comprometer las expectativas de las generaciones futuras. Si bien en este campo laboral el Trabajo Social también ha tenido participación, ello no ha ocurrido sobre la base de su especificidad en el conocimiento acerca de la equidad social y la justicia ambiental ni tampoco relevando su incursión en el ámbito de la educación social, propicia para la educación ambiental.

Un análisis de la dicotomía Naturaleza-Cultura revisitando la institucionalidad ambiental permite formular una lectura desde el Trabajo Social que interpela al derecho positivo favoreciendo el debate entre la sustentabilidad fuerte y la sustentabilidad débil. El despliegue de esta discusión deja en evidencia que el campo de la intervención socioambiental ha tenido escasa atención por parte del Trabajo Social, aunque es posible establecer que existe un interés incipiente en Sudamérica.

Palabras clave: Intervención socioambiental, institucionalidad ambiental, Trabajo Social.

Summary

One of the main features of environmental institutions is the multidisciplinary character that conceives its mission to protect the environment so as not to compromise the expectations of future generations. While this work field social work has also been involved, this has not happened on the basis of their specific knowledge about social equity and environmental justice neither recognize into the field of social education, or foster on environmental education.

An analysis of the nature-culture dichotomy revisiting environmental institutions can make a reading from Social Work that challenges to positive laws favoring sustainability debate between strong and weak sustainability. The deployment of this discussion makes it clear that the field of socio-environmental intervention has had little attention from social work, although it is possible to establish that regard there is a growing interest in South America.
\end{abstract}

Key words: Socio-enviromental intervention, environmental institutions, Social Work.

\section{Introducción}

La institucionalidad ambiental en Chile tiene un recorrido que podría encontrar entre sus primeras expresiones normativas en la Ley de corta de bosques, publicada en 1872, lo que más bien pudiera ser una continuidad respecto de los diversos antecedentes que demuestran el interés de la corona española en la regulación ambiental en tiempos coloniales (Camus, 2004 y 2014). No obstante la larga data de este interés administrativo, fue en 1994 que se registró un cambio cualitativo de marca mayor al crearse la Comisión Nacional del Medio Ambiente
(CONAMA), un organismo coordinador que comenzó a actuar como ventanilla única para una institucionalidad hasta entonces del todo dispersa, con cerca de una treintena de servicios públicos con competencia ambiental (Arellano, 2014). Este último hito fue sin duda influido por los acuerdos de la conferencia de Río en 1992 (Porras et al., 2012). No obstante, en la trayectoria reciente, la postulación de Chile a la Organización para la Cooperación y el Desarrollo Económico (OCDE; OECD por sus siglas en inglés) incidió de manera considerable en un rediseño que comenzó a gestarse en 2005, 
cuando este organismo en conjunto con la Comisión Económica para América Latina y el Caribe (CEPAL) publicaron las Evaluaciones del desempeño ambiental: Chile (OCDE/CEPAL, 2005). Por entonces se criticaban las dificultades operacionales de una institución transversal en una administración pública vertical, la incomprensión jurídica y de gestión del concepto de coordinación, un excesivo protagonismo de la autoridad política en aspectos considerados de mera competencia técnica, así como asimetrías de información. Otro aspecto sensible era apreciable un actuar vertical que debilitaba una gestión local que sufría los embates de las demandas sociales y ciudadanas pues contaba con instrumentos de gestión ambiental insuficientes para responder a las expectativas, incluyendo una fiscalización dispersa e inorgánica, circunscrita a competencias sectoriales sin capacidad de articulación (Fernández, 2012).

La tramitación de la ley 20.417 que creó el Ministerio, el Servicio de Evaluación Ambiental y la Superintendencia del Medio Ambiente rediseñó la institucionalidad ambiental creada en 1994 por la Ley de Bases Generales de Medio Ambiente (LBGMA) número 19.300, generando la extinción de la CONAMA.

Este escenario institucional es, por cierto, un campo laboral en el que el Trabajo Social no sólo tiene cabida, sino que ha llegado a desplegar acciones relevantes, no obstante encontrarse en una posición subsidiaria dada la condición multidisciplinaria que condiciona a la institucionalidad ambiental en su conjunto. La multidisciplina ha sido definida como la acción de tributación de varios campos de conocimiento a uno central o jerárquicamente concentrador de las decisiones (Karsz, 2007). En el caso de la institucionalidad ambiental, todo el peritaje científico y técnico se supedita a los marcos normativos que establece el Derecho, siendo finalmente los abogados quienes dirimen los límites de la sustentabilidad.

Es aquí que cabe destacar que el rediseño de la institucionalidad con toda claridad no implicó una reforma pues las bases conceptuales se mantuvieron completamente en su lugar, lo que queda explícito al ratificarse el literal g) del artículo segundo, acerca de la definición de Desarrollo Sustentable:

"el proceso de mejoramiento sostenido y equitativo de la calidad de vida de las personas, fundado en medidas apropiadas de conservación y protección de medio ambiente, de manera de no comprometer las expectativas de las generaciones futuras" (LBGMA, 1994, p.1).

Es evidente que esta articulación de elementos sociales, económicos y de biodiversidad proviene desde el Principio 3 de los acuerdos de la Conferencia de Río 1992, que dice: "el derecho al desarrollo debe ejercerse en forma tal que responda equitativamente a las necesidades de desarrollo y ambientales de las generaciones presentes y futuras" (ONU, 1992).

Estas definiciones son expresión de una concepción decimonónica de la naturaleza por su antropocentrismo parapetado en la dicotomía Naturaleza-Cultura, donde no existe solución de continuidad en la apreciación de la especie humana como una más de las formas de vida generada en la biósfera, inaugurando la era del Antropoceno (Steffen et al., 2011) y reconfigurando el proceso evolutivo en lo que se considera como un tiempo en que la especie humana, desde el siglo XIX de la era cristiana, ha adquirido capacidades de impacto ambiental equivalentes a las de otras fuerzas de la naturaleza, desencadenando la coevolución de la naturaleza y la cultura (Norgaard, 1984).

La contundente metáfora de Walter Benjamin justamente alude a esta situación. El ángel de la historia, nos dice, no puede ver el mundo sino como una gran catástrofe, las ruinas que deja tras de sí el viento del progreso (Benjamin, 2009). Asimismo, la lectura de la llamada Nueva Historia -que ya cuenta con más de 100 años de cultivo- nos ofrece una nueva perspectiva que libera los elementos del tiempo que el positivismo maniatara en su lógica lineal, mecanicista y jerárquica para revelar que el pasado no está fijo y prisionero en el sarcófago de las causalidades sino abierto a la revisión, las interpretaciones y el reencuadre del tiempo presente (Buck-Morss, 2011). La nueva historia, entendida según la propuesta de James Harvey Robinson (1912), atiende a los acontecimientos y estructuras sociales, científicas e intelectuales de la humanidad más allá de los meros acontecimientos políticos o económicos. Hoy en día, podemos integrar a esta complejidad la dimensión no humana de la historia ambiental y la coevolución de la biósfera en el Antropoceno.

Estas herramientas conceptuales dejan al desnudo las bases del derecho positivo y su pretensión de control de la norma sobre el mundo de la vida y explicita la posición de inconsistente de la propuesta del Desarrollo Sustentable, que ha llegado a ser calificado como un oxímoron (Redclif, 2006).

Aquí resulta de máxima pertinencia la explicación que brindan Martínez y Roca desde la economía 
ecológica, categorizando el problema de la sustentabilidad en sus dimensiones de fuerte y débil (Martínez-Alier y Roca, 2001). La sustentabilidad débil se reconoce cuando el capital natural es considerado sólo un factor productivo; esta definición es la que sustenta una hipótesis en que el capital natural es sustituible por el capital fabricado. Aquí se incuba el imaginario teórico acerca de la relación entre pobreza y degradación ambiental inflamado por los discursos malthusianos que, además, intentan conectar la capacidad de carga del hábitat con la demografía humana (Martínez-Alier y Roca, 2001, p. 388). En esta posición es que debemos situar a la institucionalidad ambiental chilena, todavía dedicada al fomento de la inversión en proyectos productivos que no internalizan los efectos ambientales de sus intervenciones.

En contraposición, la sustentabilidad fuerte se concentra en las funciones diversas e insustituibles de la física de la sustentabilidad. Esto derivado de un cuerpo teórico-conceptual que resignifica los fenómenos que se debe analizar. Un primer paso es considerar los índices físicos de impacto ambiental de la economía para valorar la Apropiación Humana de la Productividad Primaria Neta (HANPP por sus siglas en inglés) (Weisz, 2001) y evaluar la huella ecológica humana en los servicios ecosistémicos (Martínez-Alier y Roca, 2001, p. 412). En este marco conceptual, en vez de prestar atención exclusiva a los flujos de dinero, los estudios y la comprensión se dirigen a reconocer los flujos de energía y materia de la biocenosis en los que incide la especie humana. Del mismo modo, una discusión podría abrirse o incorporar las propuestas de la cuestión ecosocial (Ciorino, 2014), aunque con prevenciones respecto al giro de nacionalismo metodológico apreciable en esta lectura (Goig, 2007) o al giro ecoterritorial (Svampa, 2011).

Este otro tipo de balance de los elementos productivos requiere una revisión del problema de la equidad y, en el foco del Trabajo Social, de la desigualdad. En ello, a partir de los postulados de Vicente de Paula Faleiros (1989), podemos sostener que historia, teoría y metodología son indesligables, en tanto conforman una unidad.

De esta manera se delimita un campo laboral y de acción en que el Trabajo Social se intersecta con la institucionalidad ambiental y al que podremos denominar Intervención Socioambiental, entendida como "práctica que promueve la participación y la construcción de saberes de acción en el marco de una gobernanza cívica ambiental" (Maldonado, 2013, p. 83), cuyos alcances y limitaciones se revisarán para establecer que la incursión de profesionales del Trabajo Social ha generado contribuciones significativas, valoradas y bien recibidas en tanto la mirada transdisciplinaria es viable en una disciplina flexible, adaptativa y que cuenta con la amplia gama de herramientas que proveen tanto las ciencias sociales como las humanidades.

\section{Institucionalidad ambiental en Chile}

Entre el ahora-presente -de la década de 2010- de la institucionalidad ambiental y la convención de Río de 1992 podría haber un puente de conexión que convendrá examinar. Es el lapso entre 1994 y 2010 en Chile cuando las estructuras y funciones del Estado en la República, como se verá, tuvieron un cariz del tiempo colonial borbónico. Esta puerta de conexión entre el pasado reciente y el tiempo previo a la República es un antecedente interesante de estudiar para observar los procesos de continuidad y cambio en las lógicas y escenarios en los que el Trabajo Social tiene participación.

La CONAMA (de 1994 a 2010) fue concebida como un servicio público funcionalmente descentralizado, con personalidad jurídica y patrimonio propio, sometido a la supervigilancia del Presidente de la República a través del Ministerio Secretaría General de la Presidencia. Su máxima autoridad era el Consejo Directivo, organismo colegiado integrado por 13 ministros de Estado, siendo presidido por el Ministro Secretario General de la Presidencia, ubicándole, por tanto, como primus inter pares. Los órganos que constituían la CONAMA eran el Consejo Directivo, la Dirección Ejecutiva, el Consejo Consultivo y las Comisiones Regionales del Medio Ambiente (COREMAS).

La Dirección Ejecutiva era el órgano de CONAMA al que le correspondió implementar y ejercer las tareas encomendadas por la ley y cumplir los acuerdos y lineamientos fijados por el Consejo Directivo. El Consejo Consultivo, como su nombre lo indica, era el órgano de consulta que permitía a la CONAMA recabar opiniones y cumplir con las demás exigencias legales. Su composición era representativa de los sectores: 1. empresarial, 2. laboral, 3. de organismos no gubernamentales dedicados a la protección del medio ambiente y 4 . Universidades.

Por su parte, las COREMA eran los organismos a través de los cuales se pretendía desconcentrar el servicio en cada región administrativa del país (13 en 1994 y 15 desde 2007). Éstas eran integradas por el Intendente Regional, quien la presidía, los gobernadores y secretarios regionales ministeriales de los ministerios que participaban en el Consejo 
Directivo, cuatro consejeros regionales y el Director Regional de la CONAMA. Tenían por función desarrollar, al interior de cada región, las labores de competencia del servicio, las políticas regionales de desarrollo y de protección ambiental. Luego, cada COREMA contaba con un Comité Técnico de Evaluación, conformado por los profesionales de los servicios públicos con competencia ambiental que eran coordinados por la sede regional de la CONAMA, que sólo actuaba como ventanilla única para los distintos actores sociales implicados en el uso de los denominados Instrumentos de Gestión Ambiental: Evaluación de Impacto (SEIA), Normas Ambientales, Educación Ambiental, Fondo de Protección Ambiental y Participación Ambiental Ciudadana.

Este diseño acarreó una serie de críticas producto del nutrido contingente de autoridades políticas cuya función era representar al Poder Ejecutivo en los territorios y relegar a los municipios a una gestión de carácter técnico, además de supeditar decisiones técnicas o científicas a las contingencias políticas, lo que ponía en tensión permanente las exigencias del mercado y la industria con las expectativas de la preservación o manejo cauteloso de los ecosistemas en el ámbito de la ciudadanía y la sociedad civil en general. Afloraba así nuevamente el viejo conflicto del poder local con el metropolitano, unitario y nacional (Benítez y Salazar, 1998; Boisier, 1990). Sin embargo, uno de los elementos del diseño que no ha sido observado ni estudiado en detalle es la similitud del patrón de lógica de organización que tuvo esta Comisión con el del Real y Supremo Consejo de Indias que se constituyó como organismo asesor de la corona española desde 1524 hasta el 17 de abril de 1812 .

Los integrantes del Consejo de Indias eran su presidente, que debía reunirse todas las semanas con el rey para el trámite de consulta, informando los temas abordados por el Consejo. Contaba con 12 consejeros de indias: letrados, gente versada en ciencia jurídica y eruditos en temas americanos, ya fuera por experiencia como funcionarios en las Indias o como expertos en legislación indiana.

Además, el Consejo de Indias contaba con personal de planta: El fiscal, dos secretarios, un Secretario del Perú y otro Secretario de Nueva España, los oficiales de los secretarios, un escribano que redactaba las declaraciones de los acusados en los juicios en los tribunales, un gran canciller que custodiaba el sello real con el que se refrendaban todos los documentos oficiales del Consejo de Indias (lo que en Chile hoy en día sería un notario). El Consejo contaba, además, con relatores, contadores, un astrónomo, un cosmógrafo (que informaba los descubrimientos en el Nuevo Mundo), un cronista o guionista mayor de Indias que escribía la historia de Indias y un abogado de pobres.

Queda aquí plasmada una orgánica que despliega una lógica de administración moderna del poder absoluto (Albareda, 2012), donde se fragmentan las responsabilidades según diversos conocimientos específicos para llegar a una síntesis que debe ser reseñada al poder soberano.

El rediseño de la institucionalidad ambiental procedió a segregar sus funciones generando nuevos organismos (Lobos, 2015). A través de la ley 20.417 se extinguió la CONAMA, siendo sustituida por un Ministerio del Medio Ambiente, una Superintendencia a cargo de la fiscalización del cumplimiento de los dictámenes de un servicio público independiente que es el Servicio de Evaluación Ambiental (SEA). Además, se propuso la creación de tribunales ambientales y un Servicio de Biodiversidad y Áreas Protegidas, que aún está en estado de proyecto de ley.

La situación del SEA es la que mejor revela el proceso de continuidad en el cambio en la institucionalidad ambiental, pues la labor de este servicio público se encuentra bajo la tutela del Comité de Ministros, integrado por los ministros de Estado de las carteras de: Medio Ambiente, quien lo preside, Salud, Economía, Fomento y Turismo, Agricultura, Energía y de Minería. Este comité es el encargado de revisar las reclamaciones que se efectúen a los procesos de evaluación de impacto ambiental en caso que los eventuales afectados así lo soliciten. En definitiva, el proceso técnico continúa bajo la jurisdicción del poder político.

No obstante, las tensiones internas persisten: el SEIA como escenario de los conflictos sociales, políticos, económicos y culturales, la presión internacional por mejorar los estándares de desempeño ambiental, la continua disminución de presupuesto del Fondo de Protección Ambiental, la creación de nuevos instrumentos como la Gestión Ambiental Local (GAL), la controversial producción de conocimiento en la que los bienes públicos quedan supeditados a las lógicas del mercado (consultoras especialistas en tramitación de proyectos), la frágil labor de la Evaluación Ambiental Estratégica.

En todas estas pugnas institucionales hay trabajadores y trabajadoras sociales que cumplen distintas tareas y que bogan en un aprendizaje que se forja únicamente en el fragor de los roces de la institucionalidad con el mercado y una ciudadanía variada. 
Esta situación vista en un encuadre de Análisis Social Multicriterio exige un debate en torno a las lógicas del derecho administrativo y lo que Agamben (2010) connota al referirse al estado de la democracia: la tensión entre el poder constituyente y el poder constituido que configuran el poder soberano y que interpelan al Trabajo Social en torno a las jerarquías de sus lealtades: si la institucionalidad o la voluntad soberana popular. En esta encrucijada se encuentra el problema de la sustentabilidad.

\section{Intersecciones del TSO, la historia y la sustentabilidad}

En el mundo anglosajón existe un cierto interés por el problema de la sustentabilidad y el TSO (Kemp, 2011; Grey, Coates y Hetherington, 2012; Peeters, 2012) e incluso es posible reconocer ciertas distinciones incipientes, como la del Trabajo Social ecológico (Case, 2016).

En la América Latina es posible reconocer alguna inquietud, cuyas expresiones en el medio académico aún resultan dispersas, aunque resulta prematuro concluir al respecto, debido a que se requiere un análisis bibliométrico detallado, en tanto los conceptos de búsquedas son lo suficientemente amplios como para incluir investigaciones y problematizaciones teóricas de carácter muy distinto al campo de la sustentabilidad, medio ambiente, ecología y/o desarrollo sustentable.

No obstante lo anterior, desde una apreciación general es posible atribuir a una constitución ideológica antropocéntrica la desafección del TSO con los elementos del entorno, muy en sintonía con una cosmovisión cartesiana.

Allí se ha alojado una apreciación de la subjetividad desde el TSO que transita entre el objetivismo y el subjetivismo, que tiende a demandar una composición positivista del entramado institucional y que exhibe frecuentemente una normalización sobreaprendida donde la respuesta profesional se orienta a la satisfacción de los criterios de la institución por sobre los intereses del goce y el deseo del sujeto. Los polos de fluctuación de los profesionales van del desamparo aprendido a la subjetividad heroica (Castro, 2015; De la Aldea y Lewkovic, 2014).

De un modo definitivo, el abordaje de estos malestares profesionales, muchos de los cuales se encuentran en el silencio, requiere un tratamiento interdisciplinario. Y también demanda un análisis político de los métodos de trabajo y de articulación de la gestión profesional del oficio y la disciplina. Esas discusiones de carácter de Investigación y Desarrollo e Innovación son las que, usualmente, se dan de manera fragmentaria y no logran enlazar con fluidez los procesos con la administración de las ideas y la información.

Sumido ya en las lógicas de la complejidad, el TSO tiene suficientes bondades y atributos para asumir una participación relevante en las discusiones del problema de la Sustentabilidad, asimilando el mandato de concentrarse en la cuestión de la equidad y tensionando el programa del Desarrollo Sustentable, cuya condición de oxímoron debe ser consciente.

En ello, las contribuciones de la Historia, la Filosofía y la Literatura son valiosas, más aún cuando se propone para el TSO la capacidad de reconocer la legitimidad de la pluralidad de las formas de vida, donde suele encontrarse una fricción permanente con el mundo institucional y su acto reiterado o compulsión por la estandarización.

Son precisamente los valores de la sustentabilidad aquellos que pondrán en cuestión el ejercicio rutinario de la desigualdad, la violencia del Estado, el crecimiento económico hiperconcentrado, las sobreexigencias a los servicios ecosistémicos.

El problema de la desigualdad es justamente la materia en que se desempeña el TSO y, por lo tanto, su labor no puede prescindir de una base de lectura teórica económica. El problema es la selección de la corriente de pensamiento pertinente. Se trata de apostar por un enfoque que problematice el análisis tecnoeconómico centrado en la crematística, que no pasa de rondar en las coordenadas de la búsqueda del índice del menor costo, sea medido en economía de escala, de continuidad o de velocidad. Por lo tanto, se propone estudiar otros indicadores, como el cálculo de la HANPP ${ }^{1}$. Desde esta perspectiva parece oportuno recomendar un análisis interpretativo en busca de significaciones de lo que entendemos por relaciones económicas (Geertz, 2008).

Es el desempeño económico, pero no el meramente monetario sino el ecológico, el que es materia constitutiva de la sustentabilidad y, en esta última, se problematiza la cuestión de la inequidad no como

1 Haberl et al., op. cit. De este modo, el problema se orienta a conocer y determinar qué herramientas se utilizan para efectuar la comparación y análisis de alternativas. En todos los casos se trata de construcciones culturales que intentan objetivar criterios y, por tanto, saberes y disposiciones valóricas, pues son los argumentos que se utilizan los que entregan una clave para adentrarnos en el significado que opera en las prácticas culturales y aquella tan acertada sintesis de Michel De Certeau (2010) en La invención de lo cotidiano. 
un asunto de gestión redistributivo, sino como expresión multifacética de la violencia y, en particular, como una forma de violencia estructural.

Aquella violencia, luego institucionalizada y normalizada, es la que ubica a los sectores populares en posición de estado de excepción (Agamben, 1998, 2005 y 2008). De ahí que la propuesta de un TSO abocado a la transformación del conflicto (JiménezBautista, 2012) sea en núcleo organizador de las líneas de trabajo que se proponen a continuación para la intervención socioambiental desde el Trabajo Social.

Es por ello que, entre muchas opciones factibles de desarrollar, se pone por eje temático a la Sustentabilidad (Martínez-Alier, 2009). Es aquí donde se puede entrar con amplias libertades al debate por la equidad, ya no sólo desde el problema exclusivo de las estructuras sociales o de los efectos psicosociales sino asumiendo las materias de la contradicción de un tipo de crecimiento económico inconducente a la sustentabilidad y equilibrios de la biósfera. Se trata, ante todo, de un esfuerzo por dotar de herramientas de discusión al TSO en un ámbito temático del que ha permanecido alejado a pesar de las evidentes conexiones y labores directas que le competen en ello. El tópico de la Sustentabilidad es un campo laboral por consolidar. En varios aspectos ya existe un cuerpo profesional desempeñándose ya sea en los procesos de elaboración de líneas de base en medio humano (artículo 11 de la LBGMA) o en su evaluación de impacto ambiental, tanto como en las tareas de la educación ambiental o en la gestión ambiental local. Aspectos de interculturalidad como los que demanda el Convenio 169 de la OIT en relación con los territorios indígenas o cuestiones de soberanía alimentaria referidos a los cultivos extensivos se pueden ligar a situaciones de pobreza en las que las distintas institucionalidades presentan cometidos funcionales y generan acciones de intervención social. Caben también, en una medida distinta, las labores de consulta pública que demandan distintos instrumentos administrativos, como las evaluaciones de impacto ambiental, evaluaciones ambientales estratégicas, instrumentos de planificación territorial, planes de desarrollo comunal y regional y distintas normas ambientales y políticas públicas en torno al medio ambiente y el territorio.

En todos estos campos laborales siempre hay un eje asociado a la participación, el que bien pudiera promover una mirada en torno a la cuestión de la dicotomía comunidad/sociedad, pero que en el ámbito de la sustentabilidad abre alternativas y combinaciones conceptuales que vale la pena so- meter a escrutinio (Arellano, 2014).

Los modos de vida de la profesión y la disciplina han tendido a tomarse por vías separadas cuando la evidencia del fortalecimiento de otras profesiones demuestra la necesidad de una articulación robusta que engarce la teoría y la práctica de un modo en que se deconstruya la dicotomía teoría/práctica, cuyos efectos debilitan o inhabilitan el desarrollo del cuerpo de conocimiento del TSO. Se trata, además, de encontrar un espacio social donde se requieran aportes a la perspectiva y no una asimilación de enfoques teóricos que remitan a la ejecución de repertorios preestablecidos.

En síntesis, el TSO tiene potestad para abrir sus vínculos, abrazar nuevas posiciones, para tomar un lugar relevante en los asuntos de la contingencia, la memoria y el futuro. De esto trata la continuidad y cambio en la Historia y la Sustentabilidad, donde las personas en situación de pobreza dejan de ser unos seres deficitarios y carentes para reconocerles en la gesta de la defensa de su modo de vida, su organización autónoma y su condición de seres que hacen la Historia.

\section{La intervención socioambiental del TSO}

No cabe duda que la relación del rediseño de la institucionalidad con los conflictos socioambientales es un aspecto por explorar (Sepúlveda y Rojas, 2010). Sin embargo, algunas perspectivas iniciales han considerado el estado de situación buscando comprender las distintas dimensiones en que debe operar el control, seguimiento y fiscalización a las normas ambientales (Aliste y Urquiza, 2010).

Si bien es cierto existe una amplia gama de aproximaciones a la institucionalidad ambiental (Pizarro, 2007; Acquatela y Bárcena, 2005; Matus y otros, 2003), el Trabajo Social debe reportar una posición frente a los problemas de la sustentabilidad y las articulaciones institucionales de los actores sociales y entidades no humanas.

Una de las primeras labores hacia la configuración de un marco analítico para la intervención socioambiental en el Trabajo Social es la profundización en el estado del arte de las reflexiones de profesionales y académicos interesados en este campo.

Al respecto, en el ámbito internacional se aprecia un movimiento en aquella dirección (Ramsay \& Boddy, 2016; Smith, 2013, González, 2013; Torres 2008; Abraham y otros, 2003). En Chile, entre los acercamientos de profesionales del Trabajo Social al ámbito de la intervención socioambiental, se 
pueden destacar justamente los conflictos socioambientales hídricos (Jerez, 2015a y 2015b), mineros (Campero, 2015; Arellano, 2015a y 2015b) e institucionales (Arellano, 2014).

Estas aproximaciones permiten visualizar el interés del Trabajo Social en los problemas distributivos en tanto formas de acumulación por desposesión y el consiguiente fenómeno de injusticia ambiental. Es posible apreciar la plasticidad en la construcción de plataformas teórico-conceptuales las más de las veces pertinentes a los casos estudiados y que se pliegan a los procesos del binomio naturaleza/cultura con procedimientos ad hoc, todas iniciativas y acciones reconocibles en el desempeño de la intervención social, esta vez, con carácter ambiental y, por lo tanto, entendible como intervención socioambiental.

\section{Discusión}

En el escenario con el que el Trabajo Social interactúa y respecto del cual se deben fomentar reflexiones críticas respecto a las prácticas de intervención socioambientales se debe configurar un marco conceptual en el dominio de la sustentabilidad con una entrada desde las humanidades que favorezca la apertura y fortalecimiento de los análisis de la institucionalidad ambiental considerando sus continuidades y cambios.

Una reflexión de este alcance será atingente a las problemáticas de la gestión y transformación del conflicto socioambiental y las controversias sociotécnicas que allí se alojan. Para ello, un requisito previsible es que se desmonten las narrativas institucionalizantes que subsumen el quehacer profesional en la trama de un derecho administrativo a la vez incapaz de integrar la complejidad de los ecosistemas y orientado a la perpetuación de un modelo de crecimiento asentado en la sustentabilidad débil. Dadas esas condiciones, el Trabajo Social debiera fortalecerse en sus capacidades de realizar análisis de programa, es decir, contar con herramientas teórico-conceptuales adecuadas para comprender el sentido de la institucionalidad, conocer sus alcances y limitaciones y generar propuestas autónomas capaces de generar alternativas viables a las formas y procedimientos institucionales que contribuyen al conflicto y el ejercicio de las formas de violencia con componentes socioambientales.

En una posición como aquella, el Trabajo Social será capaz de incidir en la producción de conocimiento socioambiental y sociotécnico, desbordando los alcances de los estudios sociales de la ciencia y la tecnología (STS) por cuanto su campo de labores se halla en la intervención socioambiental, lo que demanda habilidades y conocimiento en torno a las trayectorias de los procesos de cambio cultural para su gestión y transformación; de ahí la relevancia de recurrir tanto a las ciencias sociales como a las humanidades.

En suma: el TSO requiere ampliar los marcos de análisis. El problema de la sustentabilidad requiere una contribución activa del TSO. El TSO requiere un aprendizaje de la teoría de la complejidad y tomar la palabra en el debate de la equidad social. $\mathrm{Y}$, finalmente, se debe reconocer sin ambigüedades que los valores sociales y la experticia del TSO coinciden plenamente con los principios de la sustentabilidad fuerte.

\section{Referencias}

ABRAHAM, E., TORRES, E., MONTAÑA, E., \& TORRES, L. M. (2003). "Acceso a los recursos y distribución de la población en tierras secas de Argentina: el caso de Mendoza. Aportes hacia la equidad territorial", Scripta Nova: revista electrónica de geografía y ciencias sociales, (7), 133-156.

ACQUATELLA, J. \& BÁRCENA, A. (2005). "Política Fiscal y Medio Ambiente: Bases para una Agenda Común, Santiago de Chile", Vol. 85, Libros de La Cepal, $266 \mathrm{pp}$

ALISTE, E. \& URQUIZA, A. (comp.) (2010). Medio ambiente y sociedad: conceptos, metodologias y experiencias de las ciencias sociales y humanas, Santiago, RIL Editores.

ARELLANO-ESCUDERO, N. (2014). Análisis social multicriterio: reflexiones en torno a la institucionalidad ambiental en la región de Valparaíso, Chile. Trabajo Social, (16), 175-186.

ARELlANO, N. (2015a). "Geomensura del conflicto. Cambiando la escala de reducción del impacto del Complejo Industrial de Ventanas, Puchuncaví-Quintero", Conferencia Anual COES 2015 Conflictos urbanos y territoriales: ¿desafiando la cohesión social?, 17 al 20 de noviembre del 2015 en Santiago de Chile, Universidad Diego Portales y de la Pontificia Universidad Católica de Chile.

ARELlANO, N. (2015b). "El parque industrial de Ventanas en Chile y su ángel de la historia", GT 85. Desnaturalizando la naturaleza: movimientos socioambientales, extractivismo y construcciones postneoliberales, en XI Reunión de Antropología del MERCOSUR (XI RAM), Montevideo, Uruguay, del 30 de noviembre al 4 de diciembre.

AGAMBEN, G. (1998). Homo Sacer. El poder soberano y la nuda vida, Valencia, Pre-Textos.

AGAMBEN, G. (2005). "El Testigo", en Lo que queda de Auschwitz. El archivo y el testigo. Homo Sacer III, Valencia, Pre-Textos, 13-40. 
AGAMBEN, G. (2008). El Reino y la Gloria. Por una genealogía teológica de la economía y del gobierno, Valencia, Pre-Textos.

AGAMBEN, G. (2010). "Nota preliminar sobre el concepto de la democracia", p. 11-13, traducción de Matthew Gajdowski en: Agamben, G., Badiou, A., Bensaïd, D., Brown, W., Nancy, J. L., Rancière, J., Ross, K. \& Žižek, S., Democracia, ¿en qué estado?, Buenos Aires, Prometeo libros.

ALVAREDA, J. (2012). "El debate sobre la modernidad del reformismos borbónico", HMiC: història moderna $i$ contemporània, (10), 6-19.

BENÍTEZ, J. \& SALAZAR, G. (comps.). Autonomía, espacio y gestión. El municipio cercenado, Santiago de Chile, Lom y Universidad Arcis.

BENJAMIN, W. (2009). "Sobre el concepto de historia" 129-152, en Estética y Politica, Buenos Aires, Las Cuarenta.

BOISIER, S. (1990). Territorio, estado y sociedad: reflexiones sobre descentralización y desarrollo regional en Chile, Valparaíso, Centro de Estudios y Asistencia Legislativa, Universidad Católica de Valparaíso.

BUCK-MORSS, S. (2011). Origen de la dialéctica negativa: Theodor W. Adorno, Walter Benjamin y el Instituto de Frankfurt. Buenos Aires, Eterna Cadencia.

CAMUS GAYAN, P. (2004). Los bosques y la minería del norte chico, s. XIX: un mito en la representación del paisaje chileno. Historia (Santiago), 37(2), 289-310.

CAMUS GAYAN, P. (2014). De la panacea a la tragedia. Bosques, erosión y forestación en Chile. Siglos XIX y XX. Revista de Historia Iberoamericana, 7(2), 10-19.

CAMPERO, C. (2015). "Derecho propietario como una dimensión del espacio: conflictos por usos de suelo en el sector minero en Chile", Conferencia Anual COES 2015 Conflictos urbanos y territoriales: ¿desafiando la cohesión social?, 17 al 20 de noviembre del 2015 en Santiago de Chile, Universidad Diego Portales y Pontificia Universidad Católica de Chile.

CASE, R. A. (2016). "Eco-social work and community resilience: Insights from water activism in Canada". Journal of Social Work, Ref:1468017316644695.

CASTRO-SERRANO B. (2015-2018). "Pensar el deseo y la institución con Lévinas y Deleuze. Lecturas del otro, lo político y lo social". Fondo de Ciencia y Tecnología para la Iniciación $\mathrm{N}^{\circ} 11150317$, Comisión Nacional de Investigación Científica y Tecnológica (Conicyt) Chile.

CIORINO NAVARRO, R. (2014). "La cuestión eco-social notas para un debate contemporáneo en ciencias sociales". Revista Perspectivas: notas sobre intervención y acción social, 24, 69-92.

DE CERTEAU, M. (2010). La invención de lo cotidiano, México, Universidad Iberoamericana.

DE LA ALDEA, E. (2008). "Capítulo I. La importancia de pensar la violencia en su multiplicidad", La violencia, las violencias. Reflexiones, experiencias e intervenciones, Viña del Mar, Sangría Editores, 21-42.

DE LA ALDEA, E. \& LEWKOVIC, I. (2014). "Cuidar al que cuida. Subjetividad Heroica", Buenos Aires, Editorial Los Talleres.

FALEIROS, V. DE P. (1989). A questão da metodologia do Serviço Social. Cadernos ABESS, n. 3, Cortez Editora.

FERNÁNDEZ-GROSSETETE，M. (2012). Historia sistematizada de la ley 20.417: instrumentos de gestión ambiental y otras materias, Memoria para optar a la Licenciatura en Ciencias Jurídicas y Sociales por la Universidad de Chile, $412 \mathrm{p}$

GEERTZ, C. (2003). La Interpretación de las culturas, Barcelona, Gedisa, 387 [duodécima reimpresión] traducida de: Geertz, C. (1973). The Interpretation of Cultures, Nueva York, Basic Books, Inc.

GRAY, M., COATES, J. \& HETHERINGTON, T. (2012). Environmental Social Work. Abingdon: Routledge.

GOIG, R. L. (2007). El "nacionalismo metodológico" como obstáculo en la investigación sociológica sobre migraciones internacionales. Empiria. Revista de metodología de ciencias sociales, 13, 101-120.

JEREZ, B. (2015b). Conflictos ecoterritoriales, megaproyectos de minería y centrales hidroeléctricas en los Andes patagónicos: escenarios y desafíos emergentes para el trabajo social. Intervención Social, 4, 1-8.

JEREZ, B. (2015a). (Des) colonialidades espacio-territoriales y conflictos Extractivistas en cuencas transfronterizas: el caso de la Patagonia argentino-chilena 2009-2010, Tesis para optar al grado de doctora en estudios latinoamericanos por la Universidad Nacional Autónoma de México.

JIMÉNEZ-BAUTISTA, F. (2012). "Conocer para comprender la violencia: origen, causas y realidad.", Convergencia, 19(58), 13-52.

KARSZ, S. (2007). Problematizar el trabajo social: definición, figuras, clínica [traducción, Irene Agoff, Saül Karsz], Barcelona: Gedisa.

KEMP, SUSAN (2011). "Recentring Environment in Social Work Practice: Necessity. Opportunity. Challenge". British Journal of Social Work (41), 1198-1210. Birmingham: British Association of Social Workers.

LBGMA (1994). Ley de Bases Generales del Medio Ambiente, publicada en el Diario Oficial de la República de Chile el 9 de marzo de 1994

LOBOS FERREIRA, K. (2015). Análisis de la nueva institucionales ambiental desde la reforma a la ley 19.300, tesina para el egreso de la carrera de Derecho, Universidad Católica de la Santísima Concepción.

MALDONADO, ANA (2013). "Contribución de la intervención social a la gobernanza ambiental: el caso de Québec", Portularia: Revista de Trabajo Social, (13), 77 86. 
MATUS, J. P. (2003). "Análisis dogmático del derecho penal ambiental chileno, a la luz del derecho comparado y las obligaciones contraídas por Chile en el ámbito del derecho internacional: conclusiones y propuesta legislativa fundada para una nueva protección penal del medio ambiente en Chile", Ius et Praxis, 9, 2, 11-57.

MARTÍNEZ-ALIER, J. (2009). El ecologismo de los pobres, Conflictos Ambientales y lenguajes de valores. Editorial Icaria Antrazyt.

MARTÍNEZ ALIER, J. \& ROCA, J. (2001). "El debate sobre la Sustentabilidad", Economía Ecológica y Política Ambiental, México, Fondo de Cultura Económica, $367-420$

NORGAARD, R. B. (1984). Coevolutionary development potential. Land economics, 60(2), 160-173.

ORGANIZACIÓN DE COOPERACIÓN Y DESARROLLO ECONÓMICO (OCDE) y Comisión Económica para América Latina y el Caribe (CEPAL) (2005). Evaluaciones del desempeño ambiental: Chile, Santiago de Chile, CEPAL, 246 pp.

ORGANIZACIÓN DE NACIONES UNIDAS (1992). Declaración de Río sobre el Medio Ambiente y el DesarroIlo, Departamento de Asuntos Económicos y Sociales, Nueva York.

PEETERS, J. (2012). "The place of social work in sustainable development: towards ecosocial practice", International Journal of Social Welfare, 21: 287-298. Hoboken, New Yersey: John Wiley \& Sons.

PIZARRO, R. (2007). "La Reforma Ambiental en Chile", Journal of Technology Management \& Innovation, 2, (2), 2-6.

PORRAS, I. T., MIRANDA, M., BARTON, D. N., \& CHACÓN-CASCANTE, A. (2012). De Río a Río+: Lecciones de 20 años de experiencia en servicios ambientales en Costa Rica. London, International Institute for Environment and Development, $96 \mathrm{p}$.

RAMSAY, S., \& BODDY, J. (2016). "Environmental Social Work: A Concept Analysis", British Journal of Social Work, doi: 10.1093/bjsw/bcw078.

REDCLIFT, M. (2006). "Sustainable development (1987-2005). An oxymoron comes of age", Horizontes Antropológicos, 12 (25), 65-84.

ROBINSON, J. H. (1912). New history, New York, The Macmillan Company.

SEPÚlVEDA, CLAUDIA Y ROJAS, ALEJANDRO (2010). "Conflictos ambientales y reforma ambiental en Chile: una oportunidad desaprovechada de aprendizaje institucional sobre participación ciudadana", Santiago, Revista Ambiente y Desarrollo, 24, 15-23.

SMITH, R. J. (2013). "A social worker's report from the United Nations Conference on Sustainable Development (Rio+ 20)", Social work, 58(4), 369-372.

STEFFEN, W., GRINEVALD, J., CRUTZEN, P., \& MCNEILL, J. (2011). The Anthropocene: conceptual and historical perspectives. Philosophical Transactions of the Royal Society of London A: Mathematical, Physical and Engineering Sciences, 369 (1938), 842-867.

SVAMPA, MARISTELLA (2011). "Extractivismo neodesarrollista y movimientos sociales. ¿Un giro ecoterritorial hacia nuevas alternativas?", 1, 185-218. En: Más allá del desarrollo. Fundación Rosa Luxemburg/Abya Yala. Primera Edición. Quito, Ecuador.

TORRES, L. M. (2008) "Hilos de agua, lazos de sangre: enfrentando la escasez en el desierto de Lavalle (Mendoza, Argentina)", Revista Ecosistemas, 17(1), 46-59.

WEISZ, HELGA; MARINA FISCHER-KOWALSKI, CLEMENS M. GRÜNBÜHEL ET AL. (2001). "Global environmental change and historical transitions". Innovation. The European Journal of Social Science Research 14 (2), 117-142. Victoria, Seychelles, United Kingdom. 